\title{
Definición y clasificación de los eventos adversos en ortodoncia, desde la perspectiva de la norma técnica Gestión del riesgo ISO 31000*
}

Definition and classification of adverse events in orthodontics, from the technical standard Risk management ISO 31000 perspective

Recibido: 11 de febrero de 2014 Revisado: 13 de abril de 2014 Aceptado: 27 de junio de 2014

Martha Patricia Castañeda Villamizar***

Fundación Centro de Investigación y Estudios Odontológicos - CIEO

Aitza Vivian Castañeda Solano****

Instituto Colombiano de Normas Técnicas y Certificación - ICONTEC

\section{RESUMEN}

La seguridad del paciente ha cobrado gran importancia durante los últimos años, en razón a que los daños causados durante la prestación de servicios asistenciales impactan la calidad de vida de las personas y generan sobrecostos en los sistemas de salud. Estos daños pueden ser prevenidos dependiendo de la importancia que las instituciones y sus profesionales den a la gestión del riesgo. El reporte de los eventos adversos en áreas ambulatorias como la odontología debe cobrar mayor importancia, ya que el gran número de pacientes y la

* Artículo de investigación.

** Correspondencia: Martha Patricia Castañeda Villamizar. Odontóloga, Especialista en Auditoria Clínica, MSC en Calidad y Gestión Integral. Consultora- Gerente Administrativa IPS. Correo electrónico: marthapcastaneda@gmail.com

***Aitza Vivian Castañeda Solano. Enfermera. Especialista en Gerencia y Auditoria de la Calidad en Salud. MSC en Calidad y Gestión Integral. Consultora. Docente Externa ICONTEC. Correo electrónico: aitzavivian@hotmail.com 
extensa duración de los tratamientos exponen a los pacientes a la materialización de un riesgo, representado en un evento adverso. El presente estudio determina los eventos adversos en ortodoncia desde la perspectiva de la norma técnica ISO 31000 (ICONTEC, 2011) y proporciona un listado que facilita su identificación y sirve para estimular el reporte y promover la gestión del riesgo durante el tratamiento de ortodoncia. Se utilizó la técnica de grupos focales, con 4 grupos de expertos. La información fue codificada cualitativamente, con saturación de categorías y reducción de ítems. La clasificación de los riesgos se realizó según la International Classification for Patient Safety 1.0 de la Alianza mundial para la seguridad del paciente de la OMS, adaptada a Colombia. Se evidenció el desconocimiento de los conceptos de riesgo y evento adverso entre los profesionales de ortodoncia, así como de un sistema de gestión. Se consolidó un listado preliminar de 154 eventos adversos que se clasificaron en 24 categorías.

Palabras clave: evento adverso, gestión del riesgo, ISO 31000, ortodoncia, seguridad del paciente.

\section{ABSTRACT}

Patient safety has become very important in recent years, due to damages caused during the provision of care services which affect the peoples's quality of life and generate cost overruns in health systems. Such damage can be prevented depending on the importance of institutions and professionals give risk management.

Most damages can be prevented depending on the interest that institutions and health professionals put on risk management. The reporting of adverse events in ambulatory settings as dentistry must have greater significance, since the large number of patients and duration of treatment exposes these patients to the materialization of a risk, represented in an adverse event. This study identifies the adverse events in orthodontics from the perspective of the technical standard ISO 31000 and provides a list for easy identification and reporting that serves to stimulate and promote the risk management reporting during orthodontic treatment. The technique of focus groups, with 4 groups of experts was used. The information was encoded qualitatively, with saturation of categories and items reduction. The WHO International Classification for Patient Safety 1.0 adapted to Colombia was used. A risk management guideline for orthodontics was done. Lack of knowledge of the concepts of risk and adverse events between the professionals of orthodontics, as well as its management from the perspective of a management system was evident. A preliminary list of 154 adverse events, classified into 24 categories was consolidated.

Keywords: Adverse event, risk management, ISO 31000 , orthodontics, patient safety.

\section{INTRODUCCIÓN}

La seguridad del paciente en los servicios de salud es una preocupación que viene desde la década de los años cincuenta del siglo pasado. En el 2000 el Instituto de Medicina de los Estados Unidos (IOM) publica el libro To err is Human: building a safer health system, el cual reporta que en Estados Unidos hasta 98.000 personas morían por año a causa de errores que podían prevenirse. A raíz de estos datos, la seguridad del paciente se convierte en una prioridad para el sistema sanitario de ese país. Posteriormente, la "Seguridad del Paciente" tomo dimensiones mundiales de gran importancia, que llevo a diferentes países a realizar estudios para conocer su situación frente al evento adverso 
en la atención en salud (Department of Health UK, 2000; National Patient Safety Agency, 2012; Aranaz, Aibar y Vitaller, 2006). La 55a Asamblea Mundial de la Salud (2002) insta a los estados miembros a que presten la mayor atención posible al problema de la seguridad del paciente y consoliden sistemas necesarios para mejorar la seguridad del paciente y la calidad de la atención sanitaria, en particular la vigilancia de los medicamentos, el equipo médico y la tecnología y la 57a Asamblea Mundial de la Salud (2004) propone formar una Alianza Internacional para la Seguridad del Paciente (World Alliance for Patient Safety) como una iniciativa global. Estas alianzas han enfocado sus acciones hacia la definición de políticas mundiales, dirigidas a los sistemas de salud y orientadas a la generación de calidad en la atención sanitaria que se brinda a los usuarios (European Commission DG Health and Consumer Protection, 2005).

En Colombia, el Ministerio de la Protección Social (2009) realiza diferentes estudios que incluyen información en materia de efectividad, seguridad y experiencia de atención en servicios hospitalarios, lo que condujo a consolidar el Sistema de Información para la Calidad, el cual incluye indicadores de calidad que incentivan el reporte de los eventos adversos y la gestión de los mismos (Aranaz y Aibar, 2010). No obstante, la evaluación y seguimiento de los eventos adversos, ha sido dirigida al ámbito médico y hospitalario, dejando a un lado el área ambulatoria, dentro de la cual se encuentra la odontología, que cuenta con más de 8 especialidades.

Según Baker, Norton, Flintoft, et ál. (2004), algunos estudios sobre eventos adversos realizados en países como Canadá mencionan la presentación de eventos adversos en odontología en un 3,6\% y en Colombia un estudio de casos realizado por FEPASDE derivado de las demandas a odontólogos muestra cómo el evento adverso es la principal causa de estos daños (Manrique,
2005). Hoy en día se hace evidente la poca información existente sobre lo que se puede considerar "evento adverso en ortodoncia" que permita manejar un lenguaje universal, operativo y estandarizado en relación con los eventos adversos, asimismo, se cuenta con poca información sobre la gestión eficaz del riesgo dentro de la gestión de calidad de las instituciones odontológicas.

Dentro de los objetivos de los lineamientos para la implementación de la Política de Seguridad del Paciente en Colombia, se contempla la prevención de la ocurrencia de los eventos adversos mediante la adopción de herramientas prácticas y se define el Sistema de Gestión del Evento Adverso (Ministerio de la Protección Social, 2008). Este estudio establece el contexto de la seguridad del paciente en el área de ortodoncia, en relación con los eventos adversos y la gestión del riesgo; determina y clasifica los eventos adversos en ortodoncia desde la perspectiva de la norma técnica ISO 31000 en las etapas de: identificar el riesgo; realizar el análisis del riesgo por medio de la consideración de las causas, fuentes, consecuencias y su probabilidad; evaluar el riesgo con el propósito de facilitar la toma de decisiones basada en los resultados del análisis realizado y finalmente, tratar el riesgo.

\section{METODOLOGÍA}

La metodología se desarrolló en torno a la perspectiva del enfoque genérico que describe la norma técnica ISO 31000, la cual proporciona directrices definidas en su marco de referencia y en el proceso de gestión del riesgo. El enfoque de la Investigación es cualitativo y se desarrolló siguiendo las fases planteadas por Hernández Sampieri, Fernández y Baptista (1997).

Previo consentimiento informado, se seleccionó una muestra no probabilística de expertos que demostraran trayectoria académica y clínica con más de 4 años 
de experiencia, con pregrado en Odontología General y Postgrado en Ortodoncia, que ejerzan o hayan ejercido como tutores de los trabajos de investigación en el postgrado de Ortodoncia y que demostraran permanente actualización de conocimientos de una Institución que presta servicios educativos y asistenciales de tipo ambulatorio en el área de la ortodoncia de la ciudad de Bogotá.

La recolección de los datos se realizó mediante el método o técnica de Grupos de Enfoque mediante el uso de filmaciones y grabaciones de voz durante todas las sesiones. Se ensamblaron 4 grupos focales distribuidos de la siguiente forma: 2 grupos focales conformados por 5 expertos cada uno, correspondientes a la Clínicas A o Clínica Autoligado y a la Clínica C o Clínica MBT. El tercer grupo focal estuvo conformado por 6 expertos correspondientes a la Clínica B o Estándar y el último grupo conformado por 4 expertos de la Clínica de Ortodoncia. Cada grupo focal tuvo en promedio una duración de 2 horas.

La construcción del instrumento se realizó de manera que las preguntas específicas fomentaran la interacción entre los expertos y las investigadoras, la profundización en las respuestas, y se minimizó la posibilidad que las investigadoras y participantes se salieran del tema definido (Tabla 1). Se revisó la grabación de cada grupo focal y se identificaron los eventos adversos teniendo en cuenta el orden en que se realizaron las preguntas definidas en el instrumento. Se realizó la transcripción de datos verbales en texto de cada grupo focal y se registraron en una base de datos. De la información transcrita, se obtuvo un listado de eventos adversos por cada grupo focal, en el orden en el que cada experto mencionaba el evento y en el orden en el que se realizaron los grupos focales. Con la información de los 4 grupos focales, se agruparon los eventos adversos que se repetían en cada lista, consolidándose una lista de los eventos adversos.
Tabla 1. Preguntas a los grupos focales

\begin{tabular}{|c|l|}
\hline 1. & ¿Se tienen identificados los riesgos en ortodoncia? \\
\hline 2. & ¿Cómo gestiona los riesgos en su labor de ortodoncia? \\
\hline 3. & $\begin{array}{l}\text { ¿Cuáles son los eventos adversos que se presentan en } \\
\text { ortodoncia y sus principales implicaciones? }\end{array}$ \\
\hline 4. & $\begin{array}{l}\text { ¿En qué etapa de prestación del servicio se presentan } \\
\text { eventos adversos? (antes de iniciar la atención, durante o } \\
\text { posterior) }\end{array}$ \\
\hline 5. & ¿Qué se hace con el evento adverso cuando se presenta? \\
\hline 6. & $\begin{array}{l}\text { ¿Qué problemas se tienen para documentar los eventos } \\
\text { adversos y para hacer seguimiento? }\end{array}$ \\
\hline
\end{tabular}

Fuente: elaboración propia.

Con los datos se generaron categorías de eventos adversos, repitiendo la misma operación varias veces hasta que se logró la saturación de las mismas y dichas categorías se alinearon con la metodología de la norma técnica ISO 31000. Cada categoría fue definida teniendo en cuenta la frecuencia con la que aparece en los datos analizados especificando su descripción, su significado y la relación entre las categorías, y finalmente fue codificada. Posteriormente se seleccionaron los eventos adversos a partir del análisis de datos generados y mediante la adaptación de la metodología establecida en la guía GTC 45 (ICONTEC, 2010) se determinó el nivel de probabilidad, consecuencia y nivel de riesgo. Se definió la frecuencia así: frecuencia mayor, los que se repiten más de 10 veces; frecuencia intermedia, los que se repiten entre 2 y 9 veces en cada grupo focal; y frecuencia menor, los que se repiten una vez en cada grupo focal. Se comparó la distribución de eventos adversos con la adaptación para uso en Colombia de la clasificación propuesta en la International Classification for Patient Safety 1.0 de la Alianza Mundial para la Seguridad del Paciente de la OMS, el Marco Conceptual de la Clasificación Internacional para la Seguridad del Paciente, Informe Técnico Definitivo. Versión 1.1, la Clasificación de los Eventos Adversos según el National Quality Forum 
- NQF, 2011 y el Listado de Referencia de Eventos Adversos Trazadores de la Calidad de la Atención en Salud, Anexo Técnico Resolución 1446 de 2006.

Con esta información se desarrolló una guía donde se definieron las posibilidades que tiene el usuario, para tomar decisiones sobre cómo tratar un riesgo identificado. Finalmente, se realizó la validación de la guía con un experto que no hizo parte de los grupos focales.

\section{RESULTADOS}

La realización de los grupos focales permitió advertir la existencia de riesgos presentes en el proceso de atención en ortodoncia. Los participantes manifestaron gran interés en conocer el resultado del estudio, sin embargo, en ellos no estaba claro el concepto de riesgo y evento adverso y las diferencias entre éstos. Los expertos coincidieron que en su ejercicio profesional se les han presentado eventos adversos, pero no los tienen claramente identificados ni cuentan con un plan definido que les oriente qué deben hacer después de que éste se presenta. Está claro que al presentarse un daño o lesión en el paciente, éste es gestionado, y de alguna manera se le da solución y se le informa al paciente. Los expertos coinciden que no siempre se registra el evento adverso en la historia clínica del paciente y que esto depende de la gravedad del mismo. Dicha gravedad no se encuentra definida, por esto, cada profesional la define a criterio propio.

Se consolidó inicialmente una lista de 154 eventos adversos. Posteriormente se realizó reducción de ítems consolidándose en 50 categorías. Con las 50 categorías preliminares se realizó la segunda reducción de ítems (saturación de categorías) obteniéndose 26 categorías a las cuales se les realizo la tercera reducción consolidándose las 24 categorías finales de Eventos Adversos, las cuales se presentan en la Tabla 2. Al realizar la definición de los eventos adversos en ortodoncia se excluyeron tres ítems no considerados eventos adversos: el dolor, considerado como un síntoma; el atrapamiento de la pierna del operador, que no es una lesión generada al paciente y la queilitis angular, ya que es difícil establecer su causa.

Tabla 2. Listado de eventos adversos en ortodoncia según frecuencia de repetición

\begin{tabular}{|c|c|c|}
\hline & Evento adverso & $\begin{array}{c}\text { Frecuencia } \\
\text { repetición }\end{array}$ \\
\hline 1 & Laceración & 49 \\
\hline 2 & Fracturas & 15 \\
\hline 3 & Alergias & 13 \\
\hline 4 & Enfermedad Periodontal & 9 \\
\hline 5 & Ingestión de cuerpo extraño & 9 \\
\hline 6 & Quemaduras & 9 \\
\hline 7 & Infección Odontológica & 6 \\
\hline 8 & Procedimiento equivocado & 5 \\
\hline 9 & Reabsorcion patológica & 5 \\
\hline 10 & Daños de la ATM & 3 \\
\hline 11 & Descalcificación por acumulo de placa & 3 \\
\hline 12 & Movilidad dental grado 111 & 3 \\
\hline 13 & $\begin{array}{l}\text { Patologías Pulpares derivadas de } \\
\text { Ortodoncia }\end{array}$ & 3 \\
\hline 14 & Luxación de diente & 2 \\
\hline 15 & $\begin{array}{l}\text { Caída del paciente de la Unidad } \\
\text { Odontológica }\end{array}$ & 1 \\
\hline 16 & Daño de la aparatología & 1 \\
\hline 17 & Espasmo muscular & 1 \\
\hline 18 & Estomatitis por contacto & 1 \\
\hline 19 & Exposición radicular & 1 \\
\hline 20 & Falla en el tratamiento & 1 \\
\hline 21 & Indicación inadecuada de aparatología & 1 \\
\hline 22 & Obstrucción de la vía aérea & 1 \\
\hline 23 & Perdida de diente & 1 \\
\hline 24 & Perdida de espacio & 1 \\
\hline
\end{tabular}

Fuente: elaboración propia. 
La frecuencia de los eventos se presenta en la Tabla 3.

Tabla 3. Eventos adversos según la distribución de frecuencia

\begin{tabular}{|c|}
\hline $\begin{array}{l}\text { Eventos adversos que se presentaron } \\
\text { con frecuencia mayor }\end{array}$ \\
\hline Laceración \\
\hline Fracturas \\
\hline Alergias \\
\hline $\begin{array}{c}\text { Eventos adversos que se presentaron } \\
\text { con frecuencia intermedia }\end{array}$ \\
\hline Enfermedad Periodontal \\
\hline Ingestión de cuerpo extraño \\
\hline Quemaduras \\
\hline Infección Odontológica \\
\hline Procedimiento equivocado \\
\hline Reabsorción patológica \\
\hline Daños de la ATM \\
\hline Descalcificación por acumulo de placa \\
\hline Movilidad dental grado 111 \\
\hline Patologías Pulpares derivadas de Ortodoncia \\
\hline $\begin{array}{c}\text { Eventos adversos que se presentaron } \\
\text { con frecuencia menor }\end{array}$ \\
\hline Luxación de diente \\
\hline Caída del paciente de la Unidad Odontológica \\
\hline Daño de la aparatología \\
\hline Espasmo muscular \\
\hline Estomatitis por contacto \\
\hline Exposición radicular \\
\hline Falla en el tratamiento \\
\hline Indicación inadecuada de aparatología \\
\hline Obstrucción de la vía aérea \\
\hline Perdida de diente \\
\hline
\end{tabular}

Fuente: elaboración propia.
Con los 24 eventos adversos definidos se realizó la clasificación en las 14 categorías (tipos) de la clasificación de eventos adversos para uso en Colombia, encontrándose que en los tipo 5 "Relacionada con la medicación o la administración de líquidos parenterales", 6 "Relacionada con la sangre y sus derivados", 7 "Relacionada con la dispensación de dieta 0 alimentos", 8 "Relacionada con la administración de oxigeno o gases medicinales", 10 "Relacionada con el comportamiento o las creencias del paciente" y 13 "Relacionados con la infraestructura 0 el ambiente físico", no hubo ningún Evento Adverso. Así, los 24 eventos adversos en Ortodoncia solo se clasificaron en 8 categorías como se puede observar en la Tabla 4. Se encontró que un evento adverso también podía ser clasificado en más de una categoría. El mayor número de eventos adversos se clasificó en la categoría 2 "Relacionados con fallas en procedimientos asistenciales" y en la categoría 9 "Relacionada con dispositivos y equipos médicos".

Se realizó la comparación de los eventos adversos en ortodoncia con el Marco Conceptual de la Clasificación Internacional para la Seguridad del Paciente cuya taxonomía está organizada en 10 Clases superiores y cada una de ellas cuenta con sus respectivas subdivisiones. La Clase "Tipo de Incidente" es el término descriptivo que se utilizó para una categoría constituida por incidentes de naturaleza común agrupada por compartir características acordadas (Organización Mundial de la Salud, 2006). De las 13 subdivisiones con las que cuenta la Clasificación Tipo de Incidente, se encontró que en 6 de ellas, (Medicación / Líquidos para administrar IV, Sangre / Productos Sanguíneos, Nutrición, Oxígeno / Gases / Vapores, Comportamiento e Infraestructura) no se clasificó ningún Evento Adverso. En las subdivisiones Administración Clínica, Proceso / Procedimiento Clínico, Documentación, Infección Asociada a la atención Sanitaria, Dispositivos / Equipos Médicos, Accidentes del Paciente y Recursos se relacionaron eventos adversos, según se observa en la Tabla 4. Se 
realizó también una comparación con la Clasificación de los eventos adversos según el National Quality Forum - NQF, 2011, que considera 7 categorías dentro de las cuales solo 6 de los 24 eventos adversos en ortodoncia pudieron ser clasificados. Así mismo la comparación con el Listado de Referencia de Eventos Adversos Trazadores de la Calidad de la Atención en Salud, Anexo Técnico Resolución 1446 de 2006 solo pudo involucrar 4 de los 24 eventos adversos definidos en ortodoncia.

Tabla 4. Clasificación de los eventos adversos en ortodoncia dentro del Marco Conceptual de la Clasificación Internacional para la Seguridad del Paciente

\begin{tabular}{|c|c|c|}
\hline Clase & Subdivisión & Eventos adversos definidos en ortodoncia \\
\hline \multirow{13}{*}{ 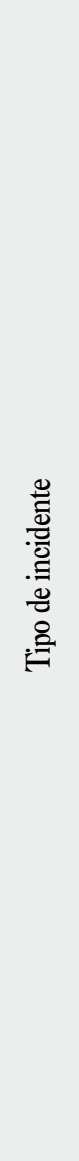 } & Administración Clínica & \\
\hline & Proceso/Procedimiento Clínico & $\begin{array}{l}\text { Fractura, indicación inadecuada de aparatología, procedimiento equivocado, luxación } \\
\text { de diente, exposición radicular, falla en tratamiento, laceración }\end{array}$ \\
\hline & Documentación & Perdida de diente \\
\hline & Infección Asociada a la atención Sanitaria & Infección odontológica, estomatitis por contacto \\
\hline & Medicación/Líquidos para administrar IV & \\
\hline & Sangre / Productos Sanguíneos & \\
\hline & Nutrición & \\
\hline & Oxígeno / Gases / Vapores & \\
\hline & Dispositivos / Equipos Médicos & $\begin{array}{l}\text { Alergias, Daño de aparatología, daño en ATM, espasmos muscular, estomatitis } \\
\text { por contacto, indicación inadecuada de aparatología, ingestión de cuerpo extraño, } \\
\text { laceración, luxación de diente, quemadura, obstrucción de la vía aérea, descalcificación } \\
\text { por acumulación de placa. }\end{array}$ \\
\hline & Comportamiento & \\
\hline & Accidentes del Paciente & $\begin{array}{l}\text { Caída del paciente de la unidad, descalcificación por acumulo de placa, espasmos } \\
\text { muscular, estomatitis por contacto, fracturas, laceración, luxación de diente, movilidad } \\
\text { dental grado lll, obstrucción de la vía aérea, patología pulpares derivadas de la } \\
\text { ortodoncia, perdida de diente, perdida de espacio, reabsorción patológica, quemaduras }\end{array}$ \\
\hline & Infraestructura & \\
\hline & Recursos & $\begin{array}{l}\text { Enfermedad periodontal, movilidad dental grado lll, patologías pulpares derivadas de } \\
\text { la ortodoncia }\end{array}$ \\
\hline
\end{tabular}

\section{DISCUSIÓN Y CONCLUSIONES}

Si bien la muestra de expertos seleccionada para participar en la investigación cumplía con los requisitos establecidos se evidencio desconocimiento de los expertos en la identificación y gestión del evento adverso. Con la ejecución del método de Grupos Focales, se obtuvo información con la cual se logró establecer y definir 24 categorías de eventos adversos que se presentan durante la atención del paciente en la consulta de ortodoncia.

En la comparación de los modelos de clasificación de los eventos adversos, los lineamientos para la Política de Seguridad del Paciente, Colombia adaptó la 
propuesta de la Interrnational Classification for Patient Safety, versión 1.0, que ofrece un detallado Marco Conceptual de la Clasificación Internacional para la Seguridad del Paciente (CISP). Tales lineamientos definen en Colombia una clasificación desactualizada frente a la directriz mundial, sin embargo, ésta clasificación colombiana es la que más permitió ajuste de los eventos adversos en ortodoncia definidos en la investigación.

Los eventos adversos en ortodoncia definidos en la presente investigación pudieron ser ubicados en algunas clasificaciones internacionales tales como la definida en el Marco Conceptual de la Clasificación Internacional para la Seguridad del Paciente. Contrario a lo anterior, la clasificación de los eventos adversos según el National Quality Forum - NQF, 2011 es específica para eventos adversos de tipo hospitalario, por tanto, muy excluyente de los eventos adversos de tipo ambulatorio, incluidos los de ortodoncia.

El listado de referencia en Colombia de Eventos Adversos Trazadores de la Calidad de la Atención en Salud del Anexo Técnico Resolución 1446 de 2006 es limitado y aunque define eventos adversos de referencia para el reporte de las notificaciones obligatorias ante los Entes de Vigilancia y Control no incluye aquellos que se pueden presentar en áreas ambulatorias como es el caso de la ortodoncia.

Se puede inferir que si bien el enfoque de la Política de Seguridad del Paciente como parte del Sistema Obligatorio de Garantía de la Calidad en Salud de Colombia está direccionado a la prestación de una atención segura, los Sistemas (Habilitación, Acreditación y de Información para la Calidad) aún no se encuentran integrados en su totalidad, lo que se hace evidente con la poca inclusión de los servicios ambulatorios como la ortodoncia, en los listados de referencia para la notificación de eventos adversos definidos por la Resolución
1446 de 2006 ,al igual que con la falta de diseño de guías de buenas prácticas para la seguridad de la atención en esta especialidad.

Existen diferentes modelos para realizar la gestión de los eventos adversos presentados a nivel hospitalario y por supuesto ambulatorio, sin embargo, el enfoque de la gestión del riesgo que ofrece la norma técnica ISO 31000 permite el desarrollo de todo el ciclo de gestión el cual incluye no solo la valoración del riesgo sino la definición del Marco de Referencia que permite el involucramiento desde la planeación estratégica de la alta dirección hasta el establecimiento del contexto tanto interno como externo que otros modelos no involucran.

La sola definición y clasificación de los eventos adversos en ortodoncia, no permite la gestión de los mismos por parte de los ortodoncistas o interesados. La falta de definición y clasificación de los eventos adversos y la ausencia de una herramienta práctica que permita la identificación dificultan la adecuada gestión de los mismos. La formulación de una guía para gestionar el riesgo contribuye en la seguridad del paciente al establecer parámetros de referencia con base en criterios estandarizados que orientan la gestión del riesgo en ortodoncia. Conocer los eventos adversos en ortodoncia y contar con una guía que permita su gestión, facilita el cambio de cultura hacia la seguridad del paciente.

En conclusión, al determinar y clasificar los eventos adversos en ortodoncia se facilita la unificación de conceptos y criterios por parte de los ortodoncistas para tomarlos como base en una posible gestión de los mismos. Esto permite detectar la necesidad de fortalecer programas enfocados a la Seguridad del Paciente desde los ámbitos académicos y de prestación de servicios en la especialidad de ortodoncia. Es necesaria la construcción y diseño de herramientas de fácil aplicación y validadas por los mismos expertos, con el fin de 
avanzar hacia una adecuada gestión y su inseparable aprendizaje organizacional. La guía propuesta permitirá la gestión de los eventos adversos en ortodoncia al involucrar a los profesionales de la salud (en este caso, ortodoncistas) hacia la participación y confianza sobre la generación de estrategias para evitar o disminuir la materialización de los eventos adversos, fomentar el aprendizaje organizacional mediante la comunicación y la consulta, facilitar el sistema de registro e información, contribuyendo esto con la gestión del riesgo, gestionar el riesgo con la participación del paciente contribuyendo este con su autocuidado y prevención y proveer al usuario de una amplia información relacionada con los riesgos y su posible manejo.

\section{RECOMENDACIONES}

Con la definición de los eventos adversos en ortodoncia se facilita el diseño y desarrollo de herramientas prácticas que fomenten la notificación y registro de los eventos adversos presentados durante el proceso de atención del paciente, esto permitiría el fortalecimiento de los Sistemas de Información de los prestadores de servicios de salud en ortodoncia, con los cuales se favorece el aprendizaje organizacional y la toma de decisiones para la mejora.

Teniendo en cuenta que la investigación se centró específicamente en la fase de atención (durante la consulta) de ortodoncia, se considera de gran importancia realizar la definición y clasificación de los eventos adversos con su respectiva gestión de riesgos en los momentos antes y después de la consulta, con lo cual se fortalecería la Seguridad del Paciente en todo el ciclo de atención en ortodoncia.

Así mismo, la identificación del tratamiento del riesgo, dicho de otro modo, la definición de barreras para prevenir y planes de acción para tratar cada uno de los eventos adversos en ortodoncia identificados, puede complementar la gestión del riesgo, por esto, se sugiere la continuidad de la investigación haciendo uso de igual o similar metodología, para que sean los mismos ortodoncistas quienes participen en dicha construcción.

La implementación de la guía para la gestión del riesgo en ortodoncia en todo caso será de más fácil aplicación, si se cuenta con conocimientos previos de sistemas de gestión, mejoramiento continuo, seguridad del paciente, gestión del riesgo entre otros.

\section{REFERENCIAS}

Aranaz, J., Aibar, C., Vitaller, J. (2006). Estudio Nacional sobre los Efectos Adversos ligados a la Hospitalización (p. 169). ENEAS 2005. Madrid: Ministerio de Sanidad y Consumo.

Aranaz, J., Aibar, C. (2010). Estudio IBEAS Prevalencia de efectos adversos en hospitales de Latinoamérica (p. 57). Madrid: Ministerio de Sanidad, Política Social e Igualdad.

Baker, R., Norton, P., Flintoft, V., et ál. The Canadian Adverse Events Study: the incidence of adverse events among hospital patients in Canada (pp.1678-1686). En: Revista CMAJ 2004; Vol 11 No 170.

Colombia. Ministerio de la Proteccion Social. (2008). Lineamientos para la implementación de la Política de Seguridad del Paciente (p. 12). Bogota: el Ministerio.

Colombia. Ministerio de la Proteccion Social. (2009). Primer Informe Nacional de Calidad de la Atención en Salud "Incas Colombia 2009". Bogota D.C.: Ministerio de la Proteccion Social. 
Committee on Quality of Health Care in America, Institute of Medicine. (2000). To Err Is Human: Building a Safer Health System (p. 280). Washington: The National Academies Press.

Department of Health UK. (2000). An organisation with a memory. Report of an expert group on learning from adverse events in the NHS (p. 93). London: The Stationery Office.

European Commission DG. Health and Consumer Protection. (2005). Patient Safety - Making it Happen! (p. 2). Luxembourg: la Comission.

Hernández Sampieri, R., Fernández, C. y Baptista, P. (1997). Metodología de la Investigación (p. 505). México: McGraw Hill.

Instituto Colombiano de Normas Técnicas y Certificación, ICONTEC. (2010). Guía para la identificación de los peligros y la valoración de los riesgos en seguridad y salud ocupacional. GTC 45 (p. 32). Bogotá D.C.: el Instituto.
Instituto Colombiano de Normas Técnicas y Certificación, ICONTEC. (2011). Gestión del riesgo. Principios y directrices. NTC-ISO 31000 (p. 29). Bogotá D.C.: el Instituto.

Manrique, J. (2005). Comportamiento de los proceso éticos en Odontología y Casuística del FEPASDE (Vol. 11, pp. 18-23). En: Revista Medico Legal.

National Patient Safety Agency. (2012). Recuperado de http://www.npsa.nhs.uk/

Organización Mundial de la Salud. (2002). 55 Asamblea Mundial de la Salud (p. 14). Ginebra: la Organización.

Organización Mundial de la Salud. (2004). 57 Asamblea Mundial de la Salud, Resoluciones y decisiones (p. 124). Ginebra: la Organización.

Organización Mundial de la Salud. (2006). Marco Conceptual de la Clasificación internacional para la Seguridad del Paciente, Versión 1.1 (p. 9). Informe Técnico Definitivo. Ginebra. 